NASA/TM-2005-213416

\title{
Acoustic Source Modeling for High Speed Air Jets
}

Marvin E. Goldstein

Glenn Research Center, Cleveland, Ohio

Abbas Khavaran

QSS Group, Inc., Cleveland, Ohio 
Since its founding, NASA has been dedicated to the advancement of aeronautics and space science. The NASA Scientific and Technical Information (STI) Program Office plays a key part in helping NASA maintain this important role.

The NASA STI Program Office is operated by Langley Research Center, the Lead Center for NASA's scientific and technical information. The NASA STI Program Office provides access to the NASA STI Database, the largest collection of aeronautical and space science STI in the world. The Program Office is also NASA's institutional mechanism for disseminating the results of its research and development activities. These results are published by NASA in the NASA STI Report Series, which includes the following report types:

- TECHNICAL PUBLICATION. Reports of completed research or a major significant phase of research that present the results of NASA programs and include extensive data or theoretical analysis. Includes compilations of significant scientific and technical data and information deemed to be of continuing reference value. NASA's counterpart of peerreviewed formal professional papers but has less stringent limitations on manuscript length and extent of graphic presentations.

- TECHNICAL MEMORANDUM. Scientific and technical findings that are preliminary or of specialized interest, e.g., quick release reports, working papers, and bibliographies that contain minimal annotation. Does not contain extensive analysis.

- CONTRACTOR REPORT. Scientific and technical findings by NASA-sponsored contractors and grantees.
- CONFERENCE PUBLICATION. Collected papers from scientific and technical conferences, symposia, seminars, or other meetings sponsored or cosponsored by NASA.

- SPECIAL PUBLICATION. Scientific, technical, or historical information from NASA programs, projects, and missions, often concerned with subjects having substantial public interest.

- TECHNICAL TRANSLATION. Englishlanguage translations of foreign scientific and technical material pertinent to NASA's mission.

Specialized services that complement the STI Program Office's diverse offerings include creating custom thesauri, building customized databases, organizing and publishing research results ... even providing videos.

For more information about the NASA STI Program Office, see the following:

- Access the NASA STI Program Home Page at http://www.sti.nasa.gov

- E-mail your question via the Internet to help@sti.nasa.gov

- Fax your question to the NASA Access Help Desk at 301-621-0134

- Telephone the NASA Access Help Desk at 301-621-0390

- Write to:

NASA Access Help Desk

NASA Center for AeroSpace Information 7121 Standard Drive

Hanover, MD 21076 
NASA/TM-2005-213416

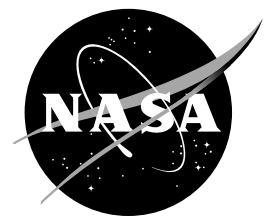

\section{Acoustic Source Modeling for High Speed Air Jets}

Marvin E. Goldstein

Glenn Research Center, Cleveland, Ohio

Abbas Khavaran

QSS Group, Inc., Cleveland, Ohio

Prepared for the

43rd Aerospace Sciences Meeting and Exhibit

sponsored by the American Institute of Aeronautics and Astronautics

Reno, Nevada, January 10-13, 2005

National Aeronautics and

Space Administration

Glenn Research Center 


\section{Acknowledgments}

The authors would like to thank Dr. Nicholas Georgiadis, nozzle branch, NASA Glenn Research Center, for providing the RANS-Wind solution to several jets discussed in this paper and Drs. Nicholas Georgiadis and John Goodrich, NASA, and Stewart Leib, Ohio Aerospace Institute, for commenting on the manuscript.

This report is a preprint of a paper intended for presentation at a conference. Because of changes that may be made before formal publication, this preprint is made available with the understanding that it will not be cited or reproduced without the permission of the author.

Available from

NASA Center for Aerospace Information 7121 Standard Drive

Hanover, MD 21076
National Technical Information Service 5285 Port Royal Road Springfield, VA 22100 


\title{
Acoustic Source Modeling for High Speed Air Jets
}

\author{
M.E. Goldstein and Abbas Khavaran \\ National Aeronautics and Space Administration \\ Glenn Research Center \\ Cleveland, Ohio 44135
}

The far field acoustic spectra at $90^{\circ}$ to the downstream axis of some typical high speed jets are calculated from two different forms of Lilley's equation combined with some recent measurements of the relevant turbulent source function. These measurements, which were limited to a single point in a low Mach number flow, were extended to other conditions with the aid of a highly developed RANS calculation. The results are compared with experimental data over a range of Mach numbers. Both forms of the analogy lead to predictions that are in excellent agreement with the experimental data at subsonic Mach numbers. The agreement is also fairly good at supersonic speeds, but the data appears to be slightly contaminated by shock-associated noise in this case.

\section{Introduction}

The acoustic analogy introduced by Lighthill ${ }^{1}$ over 50 years ago remains the principal tool for predicting the noise from high speed air jets. Its most general formulation amounts to rearranging the Navier-Stokes equations into a form that separates out the linear terms and associates them with propagation effects that can then be determined as part of the solution. The non-linear terms are treated as "known" source functions to be determined by modeling and, in more recent approaches, with some or all of the model parameters being determined from a steady RANS calculation. The "base" flow (about which the linearization is carried out) is usually assumed to be parallel and the resulting equation is usually referred to as a Lilley's ${ }^{2}$ equation.

The major drawback with these approaches is that the unsteady effects, which actually generate the sound, must be included as part of the model. This clearly puts severe demands on the modeling aspects of the prediction, which usually amount to assuming a functional form for the two-point time-delayed velocity correlation spectra. These predictions should, however, be less sensitive to the details of the model when it is possible to neglect variations in retarded time across the source correlation volume. It is therefore fortunate that this seems to be a reasonable approximation when performed in an appropriate moving frame of reference, ${ }^{3}$ assuming, of course, that the Mach number is not too large. The source models are usually tested by comparing them with measurements of the far field acoustic spectrum at $90^{\circ}$ to the downstream jet axis, which is believed to be uninfluenced by propagation effects. A major purpose of this paper is to show that this spectrum can be accurately predicted by using an appropriate acoustic analogy approach combined with some measurements of the source function that were recently carried out by Harper-Bourne. ${ }^{4}$

\section{The Acoustic Analogy Equation and its Far -Field Solution}

Reference 5 shows that the Navier-Stokes equations can be rewritten (for an ideal gas) as the Navier-Stokes equations linearized about a fictitious "base" flow but with different (in general non-linear) dependent variables, with the heat flux vector replaced by a generalized enthalpy flux and with the viscous stresses replaced by a generalized Reynolds stress. This is a true acoustic analogy (in the Lighthill ${ }^{l}$ sense) in that it shows that there is an exact analogy between the flow fluctuations in any real flow and the linear fluctuations about a fictitious "base flow" due to an externally imposed stress tensor and energy flux vector.

When the "base" flow is taken to be the unidirectional transversely sheared mean flow

$$
v_{i}=\delta_{i 1} U\left(x_{2}, x_{3}\right), \rho=\bar{\rho}\left(x_{2}, x_{3}\right), p=\bar{p}=\text { constant }
$$


where $\boldsymbol{x}=\left\{x_{1}, x_{2}, x_{3}\right\}$ is a Cartesian coordinate system, $\boldsymbol{v}=\left\{v_{1}, v_{2}, v_{3}\right\}$ denotes the velocity, $p$ the pressure and $\rho$ the density, the general equations reduce to the modified Lilley's ${ }^{1}$ equation

$$
L p_{e}^{\prime}=\frac{D}{D t}\left(\frac{\partial}{\partial x_{i}} \widetilde{c^{2}} \frac{\partial e_{i j}^{\prime}}{\partial x_{j}}\right)-\frac{\partial U}{\partial x_{i}}\left(2 \widetilde{c^{2}} \frac{\partial^{2} e_{i j}^{\prime}}{\partial x_{1} \partial x_{j}}+(\gamma-1) \frac{D^{2}}{D t^{2}} e_{1 i}^{\prime}\right)-(\gamma-1) \frac{D^{2}}{D t^{2}} \frac{\partial \eta_{j}^{\prime}}{\partial x_{j}}
$$

where

$$
L \equiv \frac{D}{D t}\left(\frac{\partial}{\partial x_{i}} \widetilde{c^{2}} \frac{\partial}{\partial x_{i}}-\frac{D^{2}}{D t^{2}}\right)-2 \frac{\partial U}{\partial x_{j}} \frac{\partial}{\partial x_{1}} \widetilde{c^{2}} \frac{\partial}{\partial x_{j}}
$$

is the variable-density Pridmore-Brown ${ }^{17}$ operator,

$$
\widetilde{c^{2}} \equiv \gamma \bar{p} / \bar{\rho}\left(x_{2}, x_{3}\right)
$$

is the square of the mean-flow sound speed, and

$$
\frac{D}{D t} \equiv \frac{\partial}{\partial t}+U \frac{\partial}{\partial x_{1}}
$$

denotes the convective derivative based on $U$. The symbol $t$ denotes the time, $\gamma$ denotes the specific heat ratio,

$$
p_{e}^{\prime} \equiv p^{\prime}+\frac{\gamma-1}{2} \rho v_{i}^{\prime} v_{i}^{\prime}
$$

is a generalized pressure fluctuation,

$$
e_{i j}^{\prime} \equiv-\rho v_{i}^{\prime} v_{j}^{\prime}+\frac{\gamma-1}{2} \delta_{i j} \rho v^{\prime 2}+\sigma_{i j}^{\prime}
$$


is the generalized stress tensor, and

$$
\eta_{i}^{\prime} \equiv-\rho v_{i}^{\prime} h_{0}^{\prime}-q_{i}^{\prime}+\sigma_{i j} v_{j}^{\prime}
$$

is the generalized stagnation enthalpy flux.

Here,

$$
\begin{gathered}
v_{i}^{\prime} \equiv v_{i}-\delta_{i 1} U, \\
h_{o}^{\prime} \equiv h^{\prime}+\frac{1}{2} v^{\prime 2},
\end{gathered}
$$

and $h^{\prime}$ denote fluctuating quantities, with $h$ being the enthalpy $\sigma_{i j}$ being the viscous stress, and $\sigma_{i j}^{\prime}$ and $q_{i}^{\prime}$ being the fluctuating viscous stress and heat flux vector respectively, which are believed to play a negligible role in the sound generation process and are therefore neglected in the following.

It is usually argued that the stagnation enthalpy flux $\eta_{i}^{\prime}$, (which corresponds to the isentropic part of the pressure density source in the Lighthill approach ${ }^{1}$ ) is only important for hot jets ${ }^{2,6-8}$ except, perhaps, at small angles to the downstream jet axis. ${ }^{9}$ It is therefore neglected in the present analysis. The resulting source function not only contains the usual quadrupole type term, but also the dipole-like term $\frac{\partial U}{\partial x_{i}}(\gamma-1) \frac{D^{2}}{D t^{2}} e_{1 i}^{\prime}$, which could have a significant effect on the predicted acoustic spectrum. The present research was initially undertaken in order to determine the appropriateness of including such a term.

This generalized Lilley's equation was solved in reference 10 in terms of the free space Green's function ${ }^{18}$ $G(\boldsymbol{x}, t \mid \boldsymbol{y}, \tau)$, which satisfies

$$
L G(\boldsymbol{x}, t \mid \boldsymbol{y}, \tau)=\delta(\boldsymbol{x}-\boldsymbol{y}) \delta(t-\tau)
$$

and has outgoing wave behavior at infinity, to obtain the following expression

$$
\overline{p^{2}\left(\boldsymbol{x}, t_{o}\right)}=\int_{-\infty}^{\infty} \iint_{V} \bar{\gamma}_{i j k l}\left(\boldsymbol{x} \mid \boldsymbol{y} ; \boldsymbol{\xi}+\hat{\mathbf{i}} U_{c} \tau_{0}, t_{o}+\tau_{o}\right) R_{i j k l}^{M}\left(\boldsymbol{y} ; \xi, \tau_{o}\right) d \boldsymbol{y} d \boldsymbol{\xi} d \tau_{o}
$$

for the pressure autocovariance ${ }^{20}$ (notice that $p_{e}^{\prime}$ reduces to $p^{\prime}$ in the far field)

$$
\overline{p^{2}}\left(\boldsymbol{x}, t_{o}\right) \equiv \frac{1}{2 T} \int_{-T}^{T} p_{e}^{\prime}(\boldsymbol{x}, t) p_{e}^{\prime}\left(\boldsymbol{x}, t+t_{o}\right) d t
$$


The symbol $V$ denotes integration over all space; $T$ denotes some large but finite time interval,

$$
\xi \equiv \boldsymbol{\eta}-\hat{\mathbf{i}} U_{c} \tau_{o}
$$

denotes a moving frame coordinate system,

$$
\bar{\gamma}_{i j k l}\left(\boldsymbol{x} \mid \boldsymbol{y} ; \boldsymbol{\eta}, t_{0}+\tau_{0}\right) \equiv \int_{-\infty}^{\infty} \gamma_{i j}\left(\boldsymbol{x}, \boldsymbol{y}, t_{1}+t_{o}+\tau_{o}\right) \gamma_{k l}\left(\boldsymbol{x} \mid \boldsymbol{y}+\boldsymbol{\eta}, t_{1}\right) d t_{1},
$$

and the propagation factor $\gamma_{k l}(\boldsymbol{x} \mid \boldsymbol{y}, t)$ is defined in reference 10. $R_{i j k l}^{M}\left(\boldsymbol{y} ; \boldsymbol{\xi}, \tau_{0}\right)$ is a moving frame correlation tensor, which is defined in terms of the fixed frame density-weighted, fourth-order, two-point, and time-delayed fluctuating velocity correlation

$$
R_{i j k l}\left(\boldsymbol{y} ; \boldsymbol{\eta}, \tau_{o}\right) \equiv \frac{1}{2 T} \int_{-T}^{T} \rho v_{i}^{\prime} v_{j}^{\prime}(\boldsymbol{y}, \tau) \rho v_{k}^{\prime} v_{l}^{\prime}\left(\boldsymbol{y}+\boldsymbol{\eta}, \tau+\tau_{o}\right) d \tau
$$

and the second order fixed frame density weighted correlation

$$
R_{i j}\left(\boldsymbol{y} ; \boldsymbol{\eta}, \tau_{o}\right) \equiv \frac{1}{2 T} \int_{-T}^{T} \sqrt{\rho} v_{i}^{\prime}(\boldsymbol{y}, \tau) \sqrt{\rho} v_{j}^{\prime}\left(\boldsymbol{y}+\boldsymbol{\eta}, \tau+\tau_{o}\right) d \tau
$$

by

$$
R_{i j k l}^{M}\left(\boldsymbol{y} ; \boldsymbol{\xi}, \tau_{0}\right) \equiv R_{i j k l}\left(\boldsymbol{y} ; \boldsymbol{\xi}+\hat{\mathbf{i}} U_{c} \tau_{o}, \tau_{o}\right)-R_{i j}(\boldsymbol{y} ; \mathbf{0}, 0) R_{k l}\left(\boldsymbol{y}+\boldsymbol{\xi}+\hat{\mathbf{i}} U_{c} \tau_{o} ; \mathbf{0}, 0\right) .
$$

The indicated arguments refer to all three terms preceding the parentheses.

Our interest is in the far field spectrum

$$
I_{\omega}(\boldsymbol{x}) \equiv \frac{1}{2 \pi} \int_{-\infty}^{\infty} e^{i \omega t_{o}} \overline{p^{2}}\left(\boldsymbol{x}, t_{o}\right) d t_{o}
$$

which can be calculated by taking the Fourier transform of equation (12) and using the convolution theorem ${ }^{21}$ to obtain 


$$
I_{\omega}(\boldsymbol{x} \mid \boldsymbol{y})=2 \pi \int_{-\infty V}^{\infty} \int_{i j} \Gamma_{i j}(\boldsymbol{x} \mid \boldsymbol{y} ; \omega) \Gamma_{k l}^{*}\left(\boldsymbol{x} \mid \boldsymbol{y}+\xi+\hat{\boldsymbol{i}} U_{c} \tau_{o} ; \omega\right) e^{-i \omega \tau_{o}} R_{i j k l}^{M}\left(\boldsymbol{y}, \xi, \tau_{o}\right) d \xi d \tau_{o}
$$

where

$$
\Gamma_{i j} \equiv \frac{1}{2 \pi} \int_{-\infty} e^{i \omega(t-\tau)} \gamma_{i j}(\boldsymbol{x} \mid \boldsymbol{y}, t-\tau) d(t-\tau)
$$

is the Fourier transform of $\gamma_{i j}$ (we use capital letters to denote Fourier transform of the corresponding lower case quantity) and we introduced $I_{\omega}(\boldsymbol{x} \mid \boldsymbol{y})$, the acoustic spectrum at $\boldsymbol{x}$ due to a unit volume of turbulence at $\boldsymbol{y}$, i.e.,

$$
I_{\omega}(\boldsymbol{x})=\int_{V} I_{\omega}(\boldsymbol{x} \mid \boldsymbol{y}) d \boldsymbol{y}
$$

in order to simplify the formulas. The relevant far field expansion of $\Gamma_{i j}$ is given in reference 10 . The only approximation made up to this point is the neglect of the enthalpy and viscous source terms, but equation (20) will depend on the turbulent source correlations only through

$$
\mathcal{R}_{i j k l}\left(\boldsymbol{y}, \tau_{o}\right) \equiv \int_{V} R_{i j k l}^{M}\left(\boldsymbol{y}, \xi, \tau_{o}\right) d \xi
$$

if variations in retarded time across the correlation volume are neglected, i.e., if $\Gamma_{k l}^{*}\left(\boldsymbol{x} \mid \boldsymbol{y}+\boldsymbol{V}+\hat{\boldsymbol{i}} U_{c} \tau ; \omega\right)$ is assumed to be constant over the correlation volume. ${ }^{3}$ However, the definitions (14) and (18) imply that the integration variable in equation (23) can be changed back to $\boldsymbol{\eta}$, which means that

$$
\mathcal{R}_{i j k l}\left(\boldsymbol{y}, \tau_{o}\right) \equiv \int_{V}\left[R_{i j k l}\left(\boldsymbol{y}, \boldsymbol{O}, \tau_{o}\right)-R_{i j}(\boldsymbol{y} ; \mathbf{0}, 0) R_{k l}(\boldsymbol{y}+\boldsymbol{\eta} ; \mathbf{0}, 0)\right] d \boldsymbol{O}
$$

Equation (20) can now be rearranged into the simpler form

$$
I_{\omega}(\boldsymbol{x} \mid \boldsymbol{y}) \rightarrow\left(\frac{2 \pi}{x}\right)^{2} \frac{2 \pi \omega}{c_{\infty}} \sin \theta \bar{\Gamma}_{i j}\left(\boldsymbol{x} \mid \boldsymbol{y}_{\perp}\right) \bar{\Gamma}_{k l}^{*}\left(\boldsymbol{x} \mid \boldsymbol{y}_{\perp}\right) \Phi^{*}{ }_{i j k l}\left(\boldsymbol{y},\left(1-M_{c} \cos \theta\right) \omega\right) \text {,as } x \rightarrow \infty,
$$


where

$$
\Phi_{i j k l}(\boldsymbol{y}, \omega) \equiv \frac{1}{2 \pi} \int_{-\infty}^{\infty} e^{i \omega \tau_{o}} \mathcal{R}_{i j k l}\left(\boldsymbol{y}, \tau_{o}\right) d \tau_{o}
$$

is the spectral tensor of the source correlation and

$$
M_{c} \equiv U_{c} / c_{\infty}
$$

is the convective Mach number of the turbulence. This result shows that it is only necessary to model the overall spectral tensor itself and not the detailed two-point time-delayed correlations of the turbulence. However, the radiated sound should still be relatively insensitive to the detailed turbulence structure even when the latter quantities are modeled (as is at least partially done below). This would not be the case if the moving frame had not been introduced before neglecting the retarded time variations. ${ }^{3}$

Our interest here is in the spectrum at $90^{\circ}$ to the jet axis where $\cos \theta=0$. Reference 10 shows that

$$
\begin{aligned}
& I_{\omega}(\boldsymbol{x} \mid \boldsymbol{y})=\frac{\left(\omega / c_{\infty}\right)^{4}}{(4 \pi x)^{2}}\left[\frac{x_{i} x_{j}}{x^{2}}-\frac{\gamma-1}{2} \delta_{i j}+\frac{i(\gamma-1) \delta_{1 i}}{\omega} \frac{\partial U}{\partial y_{j}}\right]\left[\frac{x_{k} x_{l}}{x^{2}}-\frac{\gamma-1}{2} \delta_{k l}\right. \\
& \left.-\frac{i(\gamma-1)}{\omega} \delta_{1 k} \frac{\partial U}{\partial y_{l}}\right] \Phi_{i j k l}^{*}(\boldsymbol{y} ; \omega) \text { for } \theta=\pi / 2,
\end{aligned}
$$

when $\widetilde{\mathrm{c}_{0}^{2}}=\mathrm{c}_{\infty}^{2}=$ constant, i.e., in the isothermal case.

\section{The Quasi-Normal and Axisymmetric Turbulence Approximations}

To proceed further, we need to know something about the source spectral tensor $\Phi_{i j k l}$. The usual approach ${ }^{3,12,13}$ is to begin by assuming that the turbulence is quasi-normal ${ }^{16}$ (see ref. 10) in order to obtain some relations among its components. It then follows that

$$
\begin{aligned}
R_{i j k l}\left(\boldsymbol{y}, \boldsymbol{O}, \tau_{o}\right)-R_{i j}(\boldsymbol{y} ; \mathbf{0}, 0) R_{k l}(\boldsymbol{y}+\boldsymbol{\eta} ; \mathbf{0}, 0)=R_{i k}\left(\boldsymbol{y} ; \boldsymbol{\eta}, \tau_{o}\right) R_{j l}\left(\boldsymbol{y} ; \boldsymbol{\eta}, \tau_{o}\right) \\
+R_{i l}\left(\boldsymbol{y} ; \boldsymbol{\eta}, \tau_{o}\right) R_{j k}\left(\boldsymbol{y} ; \boldsymbol{\eta}, \tau_{o}\right)
\end{aligned}
$$

To further reduce the number of independent components it is usual to assume some kinematically possible symmetric form for the second order correlations. Early studies ${ }^{23}$ assumed the turbulence to be isotropic, but that turns out to be incompatible with the Harper-Bourne ${ }^{17}$ measurements that will be introduced below. The simplest assumption compatible with his results is the one introduced in references 12 and 13, namely that the turbulence is axisymmetric which implies that ${ }^{16}$ 


$$
R_{i j}\left(\boldsymbol{y} ; \boldsymbol{\eta}, \tau_{o}\right)=A_{0} \eta_{i} \eta_{j}+B_{0} \delta_{i j}+C_{0} \delta_{1 i} \delta_{1 j}+D_{0}\left(\delta_{1 j} \eta_{j}+\delta_{1 j} \eta_{i}\right)
$$

where the symbols $A_{0}, B_{0}, C_{0}$, and $D_{0}$ denote functions of $\boldsymbol{y}, \tau_{0}$, and $\eta_{\perp} \equiv \sqrt{\eta_{2}^{2}+\eta_{3}^{2}} ; A_{0}, B_{0}$ and $C_{0}$ denote even functions of the latter quantity while $D_{0}$ denotes an odd function. This model is chosen because it is the most general of those whose mathematical properties have been studied in the literature and because it reflects the fact that the cross flow velocity components tend to be much more similar to one another than to the stream-wise component (even for non-axysymmetric flows).

Inserting equation (30) into equation (29) and inserting the result into equation (25) via equations (24) and (26) yields (after a straightforward but tedious calculation that follows along the lines of the one in appendix A of ref. 12)

$$
\begin{aligned}
& I_{\omega}(\boldsymbol{x} \mid \mathbf{y})(4 \pi x)^{2} \\
& =2\left(\frac{\omega}{c_{\infty}}\right)^{4}\left[\Phi_{1}-(\gamma-1) \Phi_{2}+\left(\frac{\gamma-1}{2}\right)^{2} \Phi_{3}\right]+\left[(\gamma-1) \frac{\omega}{c_{\infty}}|\nabla M|\right]^{2} \Phi_{4},
\end{aligned}
$$

where

$$
\begin{gathered}
\Phi_{1} \equiv \frac{1}{2 \pi} \int_{-\infty}^{\infty} e^{-i \omega \tau_{o}} \int_{V} R_{22}^{2}\left(\boldsymbol{y}, \boldsymbol{\eta}, \tau_{o}\right) d \boldsymbol{\eta} d \tau_{o}, \\
\Phi_{2} \equiv \frac{1}{2 \pi} \int_{-\infty}^{\infty} e^{-i \omega \tau_{o}} \int_{V}\left(R_{23}^{2}+R_{12}^{2}+R_{22}^{2}\right) d \boldsymbol{\eta} d \tau_{o}, \\
\Phi_{3} \equiv \frac{1}{2 \pi} \int_{-\infty}^{\infty} e^{-i \omega \tau_{o}} \int_{V}\left(4 R_{12}^{2}+2 R_{23}^{2}+R_{11}^{2}+2 R_{22}^{2}\right) d \boldsymbol{\eta} d \tau_{o},
\end{gathered}
$$

and

$$
\Phi_{4} \equiv \frac{1}{2 \pi} \int_{-\infty}^{\infty} e^{-i \omega \tau_{o}} \int_{V}\left(R_{12}{ }^{2}+R_{11} R_{22}\right) d \eta d \tau_{o}
$$

are seemingly independent spectral functions. However, the coefficients $A, B, C$, and $D$ are not all independent and, when compressibility effects are neglected (i.e., when $\rho$ is treated as a constant), these turbulence correlations can be expressed in terms of two independent scalar functions of $\boldsymbol{y}, \tau_{0}, \eta_{\perp}$, and $\eta_{1}$, say $a$ and $b,,^{14,15,24}$ which scale like 


$$
b=\overline{\rho u_{1}^{2}} B\left(\tilde{\eta}_{\perp}, \tilde{\eta}_{1}\right) L_{\perp}^{2} / 2
$$

and

$$
g \equiv a-b_{\eta_{1} \eta_{1}}=\overline{\rho u_{2}^{2}} D\left(\tilde{\eta}_{\perp}, \tilde{\eta}_{1}\right)
$$

where

$$
\begin{aligned}
& \tilde{\eta}_{1} \equiv \eta_{1} / L_{1} \\
& \tilde{\eta}_{\perp} \equiv \eta_{\perp} / L_{\perp}
\end{aligned}
$$

$L_{1}$ and $L_{\perp}$ denote characteristic stream-wise and transverse length scales of the turbulence, $B$ and $G$ are $O(1)$ functions of the indicated arguments.

Turbulence measurements suggest that

$$
\varepsilon \equiv \frac{L_{\perp}}{4 L_{1}}
$$

ought to be small. In fact, Harper-Bourne's ${ }^{17}$ measurements (to be discussed below) suggest that $\varepsilon \simeq 2.7 \times 10^{-2}$. Reference 10 shows that

$$
\begin{gathered}
\frac{(4 / 3) \Phi_{1}}{2 \pi L_{1} L_{\perp}^{2}\left(\widetilde{\rho u_{1}^{2}}\right)^{2}}=\frac{\Phi_{2}}{2 \pi L_{1} L_{\perp}^{2}\left(\widetilde{\rho u_{1}^{2}}\right)^{2}}=\bar{r}^{2} \int_{-\infty}^{\infty} e^{-i \omega \tau_{0}} \int_{-\infty}^{\infty} \int_{0}^{\infty}\left(\tilde{\eta}_{\perp} \frac{\partial D}{\partial \tilde{\eta}_{\perp}}\right)^{2} \tilde{\eta}_{\perp} d \tilde{\eta}_{\perp} d \tilde{\eta}_{1} d \tau_{0} \\
\frac{\Phi_{3}}{2 \pi L L_{\perp} L_{\perp}\left(\widetilde{\rho} u_{1}^{2}\right)^{2}}=\int_{-\infty}^{\infty} e^{-i \omega \tau_{0}} \int_{-\infty}^{\infty} \int_{0}^{\infty}\left[\frac{\bar{B}^{2}}{8}+2 \bar{r}^{2}\left(\tilde{\eta}_{\perp} \frac{\partial D}{\partial \tilde{\eta}_{\perp}}\right)^{2} \tilde{\eta}_{\perp} d \tilde{\eta}_{\perp} d \tilde{\eta}_{1} d \tau_{0}\right. \\
\frac{\Phi_{4}}{2 \pi L_{1} L_{\perp}^{2}\left(\widetilde{\rho u_{1}^{2}}\right)^{2}}=\frac{\bar{r}}{2} \int_{-\infty}^{\infty} e^{-i \omega \tau_{0}} \int_{-\infty}^{\infty} \int_{0}^{\infty} \bar{B}\left(\frac{\partial}{\partial \tilde{\eta}_{\perp}} \tilde{\eta}_{\perp}^{2} D\right) d \tilde{\eta}_{\perp} d \tilde{\eta}_{1} d \tau_{0}
\end{gathered}
$$


when $O\left(\varepsilon^{2}\right)$ terms are neglected. The ratio $\bar{r}$ is defined by

$$
\bar{r} \equiv \widetilde{\rho u_{2}^{2}} / \widetilde{\rho u_{1}^{2}}
$$

Lacking any specific data to the contrary, it seems reasonable to suppose that

$$
\frac{1}{\pi} \int_{-\infty}^{\infty} e^{-i \omega \tau_{o}} \int_{V} R_{22}^{2}\left(\boldsymbol{y}, \boldsymbol{\eta}, \tau_{o}\right) d \boldsymbol{\eta} d \tau_{o}=(\Gamma \bar{r})^{2} \Phi_{0} \equiv(\Gamma \bar{r})^{2} \frac{1}{\pi} \int_{-\infty}^{\infty} e^{-i \omega \tau_{o}} \int_{V} R_{11}^{2}\left(\boldsymbol{y}, \boldsymbol{\eta}, \tau_{o}\right) d \boldsymbol{\eta} d \tau_{o}
$$

where $\Gamma$ is a constant. Equation (31) then becomes

$$
I_{\omega}(\mathbf{x} \mid \mathbf{y})\left(2 \pi x c_{\infty}\right)^{2}=C_{0}^{2} \Phi_{o}(\boldsymbol{y}, \omega)\left(\frac{\omega}{c_{\infty}}\right)^{2}\left[\omega^{2}+(\kappa|\nabla U|)^{2}\right]
$$

where

$$
2 C_{0}^{2} \equiv \frac{2}{3}(\Gamma \bar{r})^{2}\left[\frac{3}{4}-(\gamma-1)+2\left(\frac{\gamma-1}{2}\right)^{2}\right]+\frac{1}{2}\left(\frac{\gamma-1}{2}\right)^{2}
$$

is a constant, i.e., independent of $\omega$,and

$$
\begin{aligned}
& \kappa \equiv \\
& \left(\frac{\gamma-1}{2}\right) \frac{1}{C_{0}} \sqrt{\frac{2 \bar{r} \int_{-\infty}^{\infty} e^{-i \omega \tau_{0}} \int_{-\infty}^{\infty} \int_{0}^{\infty} \bar{B}\left(\frac{\partial}{\partial \tilde{\eta}_{\perp}} \tilde{\eta}_{\perp}^{2} D\right) d \tilde{\eta}_{\perp} d \tilde{\eta}_{1} d \tau_{0}}{\int_{-\infty}^{\infty} e^{-i \omega \tau_{0}} \int_{-\infty}^{\infty} \int_{0}^{\infty} \bar{B}^{2} \tilde{\eta}_{\perp} d \tilde{\eta}_{\perp} d \tilde{\eta}_{1} d \tau_{0}}}
\end{aligned}
$$

\section{The Harper-Bourne Spectrum}

The results cannot be made more explicit without inputting more specific information about the turbulence structure. This is accomplished with the aid of some recent measurements ${ }^{17}$ of the two-point fourth-order streamwise velocity correlation spectra along the centerline of the mixing layer in a low Mach number jet, which would most closely correspond to

$$
H_{o}(\boldsymbol{y}, \boldsymbol{\eta}, \omega) \equiv \frac{1}{\pi} \int_{-\infty}^{\infty} e^{-i \omega \tau_{o}} R_{11}^{2}\left(\boldsymbol{y}, \boldsymbol{\eta}, \tau_{o}\right) d \tau_{o}
$$


with the quasi-normal approximation that is being used in the present analysis.

Harper-Bourne ${ }^{17}$ divided $H_{0}$ into the three components (see his eqs. (2.5) and (2.7) on p. 2)

$$
H_{o}=H_{o}(\boldsymbol{y}, \mathbf{0}, \omega) R\left(\boldsymbol{y}, \frac{\eta_{1}}{l_{1}}, \frac{\eta_{\perp}}{l_{\perp}}, \omega\right) e^{i \omega \tau_{p}}
$$

where $l_{l}, l_{\perp}$ are the spectral stream-wise and transverse length scales (not necessarily the same as the time domain length scales $L_{1}$ and $L_{\perp}$ introduced above) and

$$
\tau_{p} \simeq \frac{\eta_{1}}{U_{c}}
$$

No assumption is made about the decomposition of the correlations into products of their space and time components with this approach.

The first factor can be evaluated from his measurements of $R_{1111}\left(\mathbf{y}, \mathbf{0}, \tau_{0}\right)$, which are reasonably well represented by the exponential $e^{-\lambda\left|\tau_{o}\right|}$. But reference 19 shows that $R_{1111}\left(\mathbf{y}, \mathbf{0}, \tau_{0}\right)$ does not in reality have a sharp cusp at $\tau_{0}=0$. A better representation would therefore be

$$
R_{1111}\left(\mathbf{y}, \mathbf{0}, \tau_{0}\right)=\frac{e^{-\lambda \tau_{0}} \operatorname{erfc} .\left(\beta-\frac{\lambda \tau_{0}}{2 \beta}\right)+e^{\lambda \tau_{0}} \operatorname{erfc} .\left(\beta+\frac{\lambda \tau_{0}}{2 \beta}\right)}{2 \text { erfc. } \beta},
$$

which behaves like $e^{-\lambda\left|\tau_{o}\right|}$ for large $\tau_{0}$ and reduces to this quantity when $\beta=0$, but smoothes out the cusp at $\tau_{0}=0$. It therefore follows that ${ }^{20}$

$$
H_{o}(\boldsymbol{y}, \mathbf{0}, \omega)=\frac{\widetilde{\lambda \rho u_{1}^{4}} e^{-(\beta \omega / \lambda)^{2}}}{\pi\left(\exp \cdot \beta^{2} \operatorname{erfc} \cdot \beta\right)\left(\lambda^{2}+\omega^{2}\right)}
$$

Inserting these into equation (46) and using the result in equations (41) and (42) shows that

$$
I_{\omega}(\mathbf{x} \mid \mathbf{y})=C_{0}^{2} \frac{\widehat{\lambda \rho u_{1}^{4}} e^{-(\beta \omega / \lambda)^{2}}\left[\omega^{2}+(\kappa|\nabla U|)^{2}\right] 2 U_{c}^{3} \bar{l}_{1} \bar{l}_{\perp}^{2} \bar{R}\left(\boldsymbol{y}, \bar{l}_{1}\right)}{x^{2} c_{\infty}^{4}\left(\exp . \beta^{2} \text { erfc. } \beta\right)\left(\lambda^{2}+\omega^{2}\right) \omega}
$$

where 


$$
\bar{R}\left(\boldsymbol{y}, \bar{l}_{1}\right) \equiv 2 \pi \int_{-\infty}^{\infty} \int_{0}^{\infty} R\left(\boldsymbol{y}, \bar{\eta}_{1}, \bar{\eta}_{\perp}\right) e^{-2 \pi i \bar{\eta}_{1} \overline{\bar{l}}_{1}} d \bar{\eta}_{1} \bar{\eta}_{\perp} d \bar{\eta}_{\perp},
$$

and $\bar{l}$ and $\bar{l}_{\perp}$ are defined by

$$
\bar{l}_{l} \equiv \frac{\omega l_{l}}{2 \pi U_{c}}
$$

and

$$
\bar{l}_{\perp} \equiv \frac{\omega l_{\perp}}{2 \pi U_{c}}
$$

Harper-Bourne obtains the best fit to his data with the non-separable form

$$
R=e^{-\sqrt{\bar{\eta}_{1}^{2}+\bar{\eta}_{\perp}^{4}}}
$$

which can be inserted into equation (50) to obtain

$$
\bar{R}=\frac{\pi}{2\left[1+\left(2 \pi \bar{l}_{1}\right)^{2}\right]^{3 / 2}} .
$$

Harper-Bourne's figure 13 shows that while $l_{1}$ and $l_{\perp}$ are constant at relatively low frequencies, it is the scaled length scales $\bar{l}_{i}$ and $\bar{l}_{\perp}$ that become constant as $\omega \rightarrow \infty$. The data is well represented by the functions

$$
\frac{\omega l_{1}}{2 \pi U_{J}} \approx \frac{1}{2}\left(1-e^{-2 S t}\right)
$$

and

$$
\frac{\omega l_{\perp}}{2 \pi U_{J}} \approx 0.15\left(1-e^{-0.5 S t}\right)
$$

where 


$$
S t \equiv \omega D_{J} / 2 \pi U_{J}
$$

and $D_{J}$ denotes the jet diameter.

\section{Extension of the Harper-Bourne Data}

Unfortunately, all of Harper-Bourne's data are taken at a single point in a very low Mach number jet, while practical interest is in much higher Mach number flows and the acoustic predictions require information about the turbulence over the entire noise producing region of the jet. We therefore attempt to extend his data by using some modeling assumptions along with the Wind code developed by NASA Glenn Research Center and the U.S.A.F. Arnold Engineering Development Center, which is a RANS code with a standard $k-\varepsilon$ turbulence model. To this end, we first assume that the time scale $\lambda^{-1}$ that appears in equation (49) is proportional to the $k-\varepsilon$ time scale $k / \varepsilon$, i.e., we put

$$
\lambda^{-1} \approx C^{\tau} k / \varepsilon
$$

where $C^{\tau}$ is an adjustable constant.

Equations (55) and (56) are extended by assuming that the time and velocity scales $D_{J} / U_{J}$ and $U_{J}$ are proportional to the $k-\varepsilon$ time and length scales $k / \varepsilon$ and $k^{1 / 2}$ respectively to obtain

$$
\frac{\omega l_{1}}{2 \pi} \approx C^{l} k^{1 / 2}\left[1-\exp \left(-C^{S} \frac{\omega k}{2 \pi \varepsilon}\right)\right]
$$

and

$$
\frac{\omega l_{\perp}}{2 \pi} \approx 0.3 C^{l} k^{1 / 2}\left[1-\exp \left(-0.25 C^{S} \frac{\omega k}{2 \pi \varepsilon}\right)\right]
$$

where $C^{l}$ and $C^{S}$ are constants. Figure 1 shows the RANS (WIND code) solution for a 2 in. diameter cold jet at the jet exit Mach number of 0.18 for which Harper-Bourne carried out his measurements. The two constants $C^{l}$ and $C^{S}$ are determined by requiring that equations (55) and (59) be in reasonable agreement with the data at the measurement point $\left(x_{1} / D_{J}=4.0, x_{2} / D_{J}=0.50\right)$. A reasonably good approximation is obtained by selecting $C^{l} \approx 3.0$ and $C^{S} \approx 0.50$. We neglected the difference in exponents between (59) and (60) but plan to remedy this oversight in the near future.

The velocity ratio $\bar{r}$ is typically close to $1 / 2 \mathrm{in}$. most cases and we shall use this value in our computations. The constant $C_{o}$ is related to the ratio $\Gamma r$ (defined implicitly by eq. (40)) by eq. (43), which for $\gamma=1.4$ becomes

$$
C_{o}^{2} \approx \frac{0.43}{3}(\Gamma r)^{2}+0.01
$$


Unfortunately, Harper- Bourne only measured the stream-wise and not the transverse velocity correlations so that $\Gamma$ is essentially unknown. But if we assume, as in reference 10 , that $B / 2=D=\bar{a}\left(\bar{\eta}_{1}, \tau_{o}\right) e^{-\bar{\eta}_{\perp}^{2} / 2}$ then this quantity is equal to $\sqrt{3}$ and it follows that

$$
C_{o}^{2} \approx 0.1472 \text {. }
$$

We treat $C^{\tau}, \beta$, and $C_{o}$ as adjustable constants, whose determination is described in the next section. It is necessary to specify the square root in equation (43) in order to fix $\kappa$. Again, Harper- Bourne does not provide enough data to ascertain this quantity, but it becomes equal to $1 \frac{1}{2}$ in. and equation (43), therefore, becomes

$$
\kappa \approx 0.1 / C_{o}
$$

when, as before, it is assumed that $\gamma=1.4$ and $B / 2=D=\bar{a}\left(\bar{\eta}_{1}, \tau_{o}\right) e^{-\bar{\eta}_{\perp}^{2} / 2}$.

\section{Comparison with Measurements}

The far-field spectra at $90^{\circ}$ to the jet axis were calculated from equations (49) and (54) at $y_{1}=100 D_{J}$ for Mach $0.50,0.90$, and 1.5 cold jets. As in the JeNo code, ${ }^{15,21}$ the local result (49) was summed over all source points within the noise producing region of the jet in order to predict the actual sound field. RANS solutions for the relevant nozzles were obtained from the WIND code with upstream boundary conditions specified in terms of the stagnation pressure and temperature at the nozzle plenum entrance. The predicted turbulent kinetic energy distribution and the corresponding time-scale for the three jets are shown in figure 2.

The calculated $90^{\circ}$ acoustic spectra are compared with the subsonic SHJAR data recently acquired at NASA Glenn Research Center and correctly expanded supersonic data obtained at Langley Research Center in figures 3 through 5. Atmospheric attenuation was removed from all measurements in order to make a lossless comparison with predictions. We expect the parameters $C^{l}$ and $C^{S}$, which were determined from the Mach 0.18 jet RANS solution, to be independent of both Reynolds number and Mach number. The adjustable constants $C^{\tau}, \beta$, and $C_{o}$ were determined by obtaining the best fit with the Mach 0.5 data. The resulting values of $C^{\tau}$ and $\beta$ turn out to be 0.35 and 0.10 respectively. The scale factor $C_{o}$ turns out to be $C_{o}^{2} \approx 0.133$, which is in remarkably good agreement with the value calculated from equation (61).

The calculated spectra are in excellent agreement with the subsonic data over the entire frequency range. The agreement is not quite as good for the supersonic case, but it is likely that this data contains a small amount of shock associated noise that is not accounted for by the theory.

Equation (63) implies that $\kappa \approx 0.27$ when $C_{o}^{2} \approx 0.133$. Figure 4 (with $\kappa \approx 0.9$ ) shows that there is very little difference between the results obtained with $\kappa$ given by this equation and those obtained with $\kappa=0$. Equation (49) shows that $I_{\omega} \sim \omega^{4}$ as $\omega \rightarrow 0$ in the latter case, which is consistent with the conventional wisdom that, at least for cold jets, the sideline noise is dominated by a quadrupole- type source as originally proposed by Lighthill.

\section{Discussion}

A simpler and, we believe, more elegant form of Lilley's equation was derived in reference 19 by introducing the new dependent variable 


$$
\pi \equiv(p / \bar{p})^{1 / \gamma}-1
$$

to obtain

$$
L \pi=\frac{D}{D t} \frac{\partial f_{i}}{\partial x_{i}}-2 \frac{\partial U}{\partial x_{j}} \frac{\partial f_{j}}{\partial x_{1}}
$$

where

$$
f_{i} \equiv-\frac{\partial}{\partial x_{j}}(1+\pi) v_{i}^{\prime} v_{j}^{\prime}-(\gamma-1) h^{\prime} \frac{\partial \pi}{\partial x_{i}}
$$

$L$ is defined by equation (3) and $v_{i}^{\prime}$ is defined by equation (9). In this context it is usual to neglect the dipole-like term $(\gamma-1) h^{\prime} \frac{\partial \pi}{\partial x_{i}}$ rather than the dipole-like term $(\gamma-1) \frac{D^{2}}{D t^{2}} \frac{\partial \eta_{j}^{\prime}}{\partial x_{j}}$ for cold air jets. It was also shown in reference 19 that this dipole-like term will not even appear in equation (66) if $\bar{c}^{2}$ is replaced by $\bar{c}^{2}+\left(c^{2}\right)^{\prime}$ (where $\left(c^{2}\right)^{\prime}=(\gamma-1) h^{\prime}$ denotes the fluctuation in the squared sound speed) in the operator $L$ defined by equation (3), so its neglect can also be interpreted to mean that the sound speed fluctuations have a negligible effect (relative to the mean) on the acoustic propagation. Since equations (2) and (65) are both exact, any differences in the predictions must be attributable to the neglect of these terms.

When the preceding analysis is applied to the present equations (i.e., equations (64) to (66) rather than equation (2)), the final result is still given by equation (41) but with $\kappa=0$, a slightly different definition of the density weighted source correlations, and

$$
C_{o}^{2}=\frac{1}{4}(\Gamma r)^{2}
$$

which becomes

$$
C_{o}^{2} \approx 0.187
$$

when the values $\Gamma$ and $\bar{r}$ obtained in the previous section are inserted-a result that is fairly close to the previous value. The principal difference between these predictions is therefore due to the second term in the factor $\left[\omega^{2}+(\kappa|\nabla U|)^{2}\right]$, which does not significantly affect the high frequency behavior of the solution but has the potential of causing $I_{\omega}$ to exhibit the dipole-like behavior

$$
I_{\omega} \sim \omega^{2} \quad \text { as } \omega \rightarrow 0
$$

in the formulation discussed in this paper rather than the quadrupole-like behavior 


$$
I_{\omega} \sim \omega^{4} \quad \text { as } \omega \rightarrow 0
$$

that always occurs when the predictions are based on equations (65) and (66). But the computations and data comparisons of the previous section show that the second term in this factor is relatively small for cold jets and that good agreement is achieved independently of whether that term is included. This is because the low frequency roll off of the acoustic spectrum is primarily determined by the peak frequency distribution of the local spectra and not by their low frequency asymptotes. We note, however, that the value of $C_{o}$ given by equation (62) is slightly closer to the "fitted" value than the one given by equation (68), but-given the uncertainty of the approximations used in the source modeling-this difference is not large enough to distinguish between these two forms of the acoustic analogy.

\section{Concluding Remarks}

The research was initially motivated by the need to distinguish between the two forms of the acoustic analogy described above. Unfortunately the results turned out to be inconclusive-with both forms of the analogy yielding excellent agreement with the data. Our hope is that similar comparisons for hot jets or jets with more complex flow fields will provide the required selectivity. But until this is done, our recommendation would be to base the jet noise predictions on the formulation (65) and (66), as was done in reference 21, since this leads to much simpler formulasespecially at angles other than $90^{\circ}$.

Finally, it is worth noting that the adjustable constant $\beta$, which measures the curvature of the temporal autocovariance at $\tau=0$, is relatively small and is therefore consistent with experimentally observed turbulence spectra. ${ }^{20}$ It is, however, somewhat puzzling that the high frequency roll off of the predicted acoustic spectra turns out to be fairly sensitive to this parameter. It is also rather unfortunate, because this quantity is difficult to measure with any accuracy.

\section{References}

${ }^{1}$ Lighthill, M.J., “On Sound Generated Aerodynamically: I. General Theory,” Proceedings R. Society Lond., A 211, 1952, pp. 564-587.

${ }^{2}$ Lilley, G.M., “On the Noise from Jets,” Noise Mechanism, AGARD-CP-131, 1974, pp. 13.1-13.12.

${ }^{3}$ Ffowcs William, J.E., “The Noise from Turbulence Convected at High Speed,” Phil. Trans. Roy. Soc., A 225, 1963, pp. 469-503.

${ }^{4}$ Harper-Bourne, M., “Jet Noise Turbulence Measurements,” AIAA Paper 2003-3214, 2003.

${ }^{5}$ Goldstein, M.E., “A Generalized Acoustic Analogy,” Journal of Fluid Mechanics, vol. 488, 2003, pp. 315-333.

${ }^{6}$ Lilley, G.M., "The Radiated Noise from Isotropic Turbulence with Applications to the Theory of Jet Noise," Journal of Sound Vibration, vol. 190, 1996, pp. 463-476.

${ }^{7}$ Morfey, C.L., Szewczyk, V.M., and Fisher, B.J., "New Scaling Laws for Hot and Cold Jet Mixing Noise Based on a Geometric Acoustics Model,” Journal of Sound and Vibration, vol. 46, no. 1, 1976, pp. 79-103.

${ }^{8}$ Lilley, G.M., "Jet Noise: Classical Theory and Experiments” in Aeroacoustics of Flight Vehicles, H. Hubbard ed., vol. 1 NASA RP-1258, and WRDC TR-90-3052, 1991, pp. 211-289.

${ }^{9}$ Freund, J.B., “Turbulent Jet Noise: Shear Noise, Self-Noise, and Other Contributions,” AIAA Paper 2002-2423, 2002.

${ }^{10}$ Goldstein, M.E., “The 90 Acoustic Spectrum of a High Speed Air Jet”, AIAA Journal, vol. 42, no. 11, Nov. 2004.

${ }^{11}$ Goldstein, M.E. and Rosenbaum, B.M., "Emission of Sound from Turbulence Converted by a Parallel Flow in the Presence of Solid Boundaries,” NASA TN D-7118, 1973.

${ }^{12}$ Goldstein, M.E. and Rosenbaum, B., "Effect of Anisotropic Turbulence on Aerodynamic Noise,” Journal of the Acoustical Society of America, vol. 54, no. 3, 1973, pp. 630-645. 
${ }^{13}$ Kerschen, E.J., “Constraints on the Invariant Function of Axisymmetric Turbulence,” AIAA Journal, vol. 21, no. 7, 1983, pp. 978-985.

${ }^{14}$ Lindborg, E., "Kinematics of Homogeneous Axisymmetric Turbulence,” Journal of Fluid Mechanics, vol. 302, 1995, pp. 179-201.

${ }^{15}$ Khavaran, A., “Role of Anisotropy in Turbulent Mixing Noise,” AIAA Journal, vol. 37, no. 7, 1999, pp. 832-841.

${ }^{16}$ Batchelor, G.K., “Theory of Homogeneous Turbulence,” Cambridge University Press, 1953.

${ }^{17}$ Pridmore-Brown, "Sound Propagation in a Fluid Flowing Through an Attenuating Duct," Journal of Fluid Mechanics, vol. 4, 1958, pp. 393-406.

${ }^{18}$ Morse, P.M. and Feshbach, H., "Methods of Theoretical Physics,” McGraw-Hill, 1953.

${ }^{19}$ Goldstein, M.E., “An exact form of Lilley's equation with a velocity quadrupole/temperature dipole source term” Journal of Fluid Mechanics, vol. 443, 2001, pp. 231-236.

${ }^{20}$ Pope, S.B., “Turbulent Flows,” Cambridge University Press, 2000, pp. 70 and 144.

${ }^{21}$ Khavaran, A., Bridges, J., and Freund, J.B., “A Parametric Study of Fine-Scale Turbulence Mixing Noise,” NASA/TM-2002-211696, 2002.

${ }^{22}$ Norum, T. NASA-Langley Jet Noise Lab Generic Nozzle Narrow Band Spectra, vol. 1, 1994 (unpublished, available electronically).
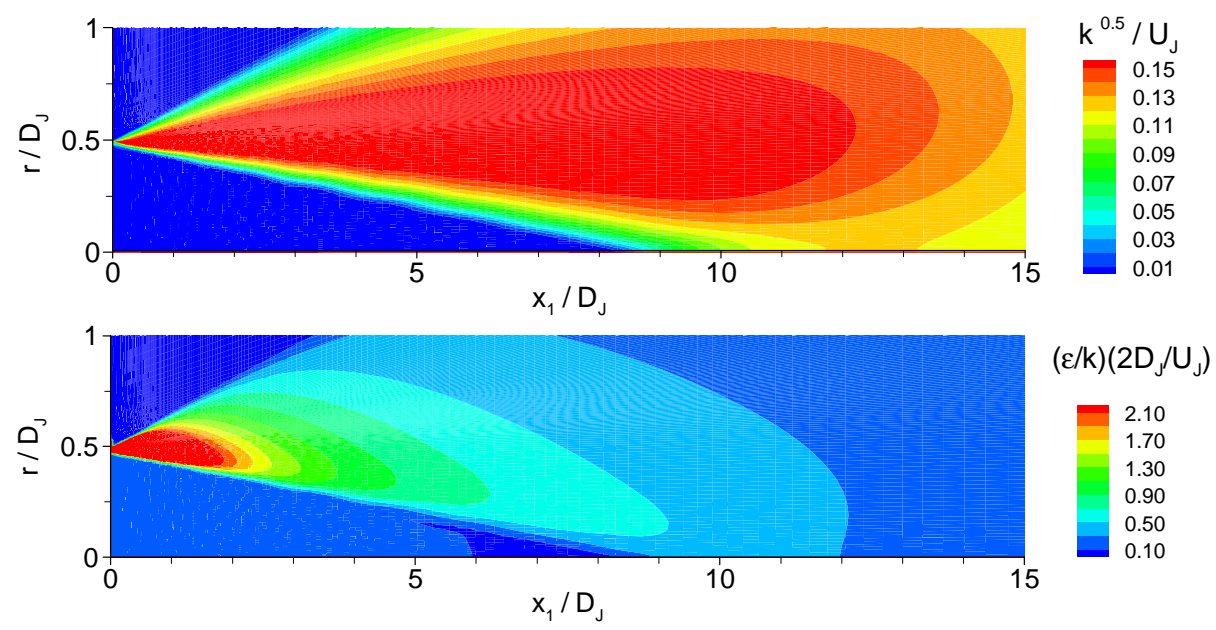

Figure 1.-Predicted turbulent kinetic energy (top), and frequency scale (bottom) in a Mach 0.18 cold jet. $r \equiv\left|\boldsymbol{y}_{\perp}\right|$. 

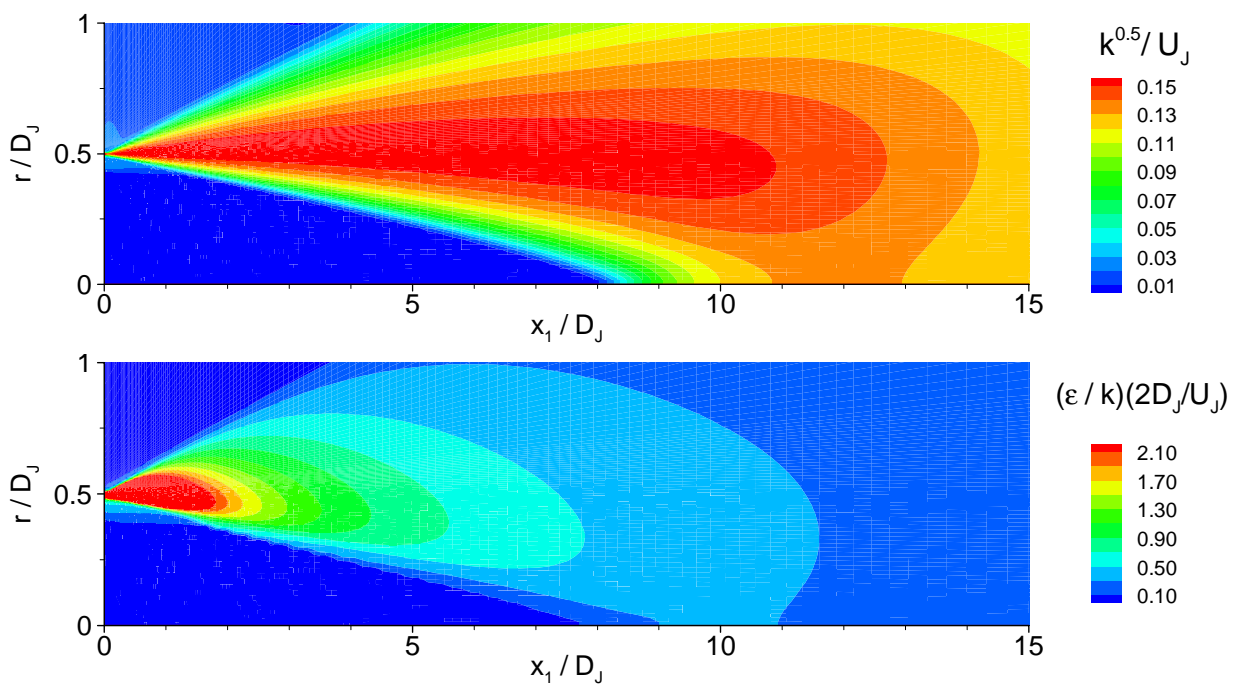

Figure 2a.-Predicted turbulent kinetic energy (top), and frequency scale (bottom) for a 2 in. diameter cold jet at Mach 0.50. $r \equiv\left|\boldsymbol{y}_{\perp}\right|$.
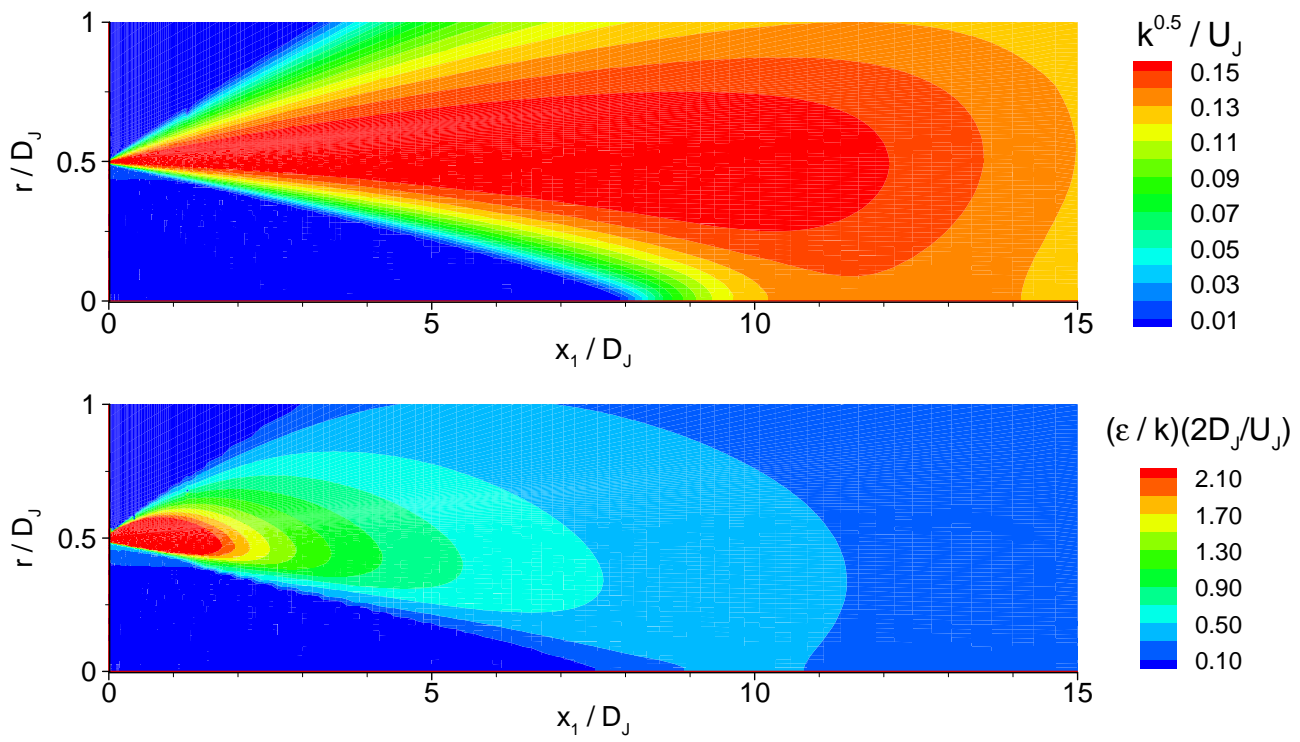

Figure 2b.-Predicted turbulent kinetic energy (top), and frequency scale (bottom) for a 2 in. diameter cold jet at Mach 0.90. $r \equiv\left|\boldsymbol{y}_{\perp}\right|$. 

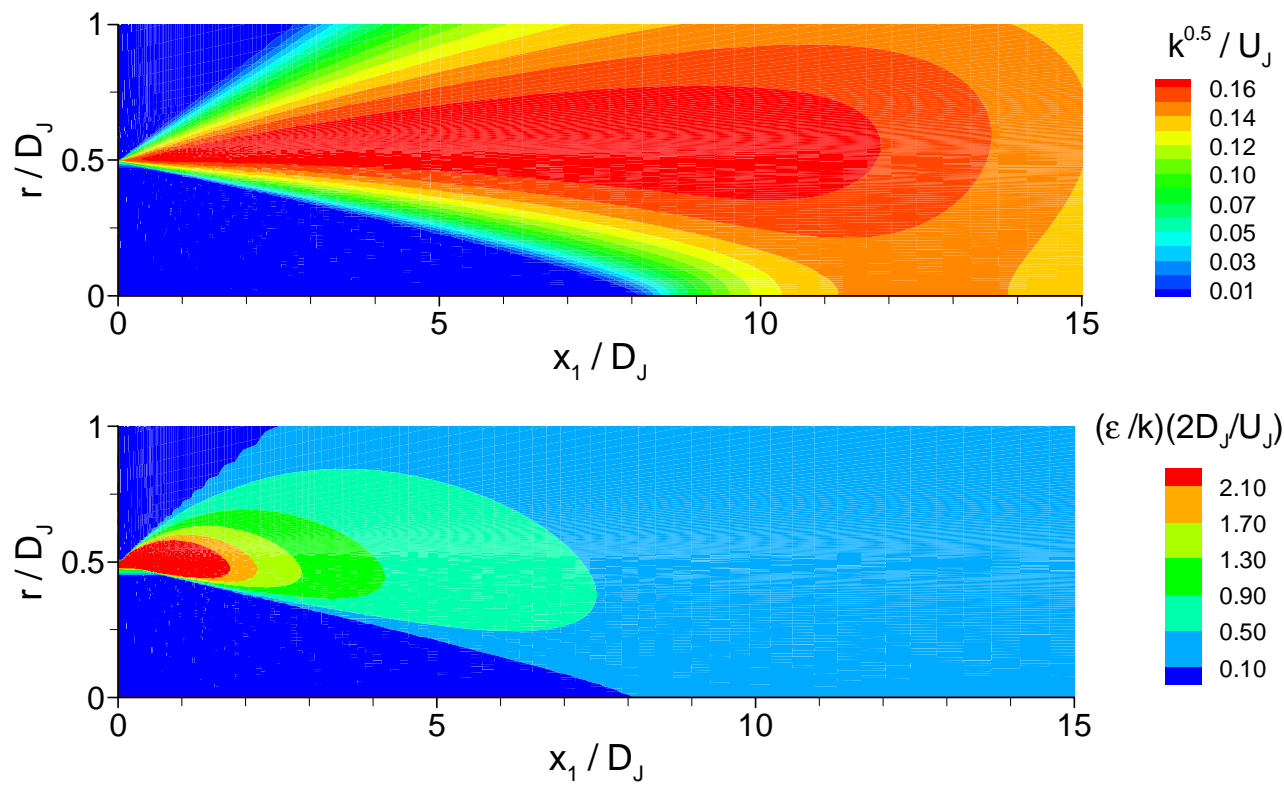

Figure 2c.-Predicted turbulent kinetic energy (top), and frequency scale (bottom) for a Mach 1.50 convergent-divergent nozzle with 1.68 in. exit diameter. $r \equiv\left|\boldsymbol{y}_{\perp}\right|$.

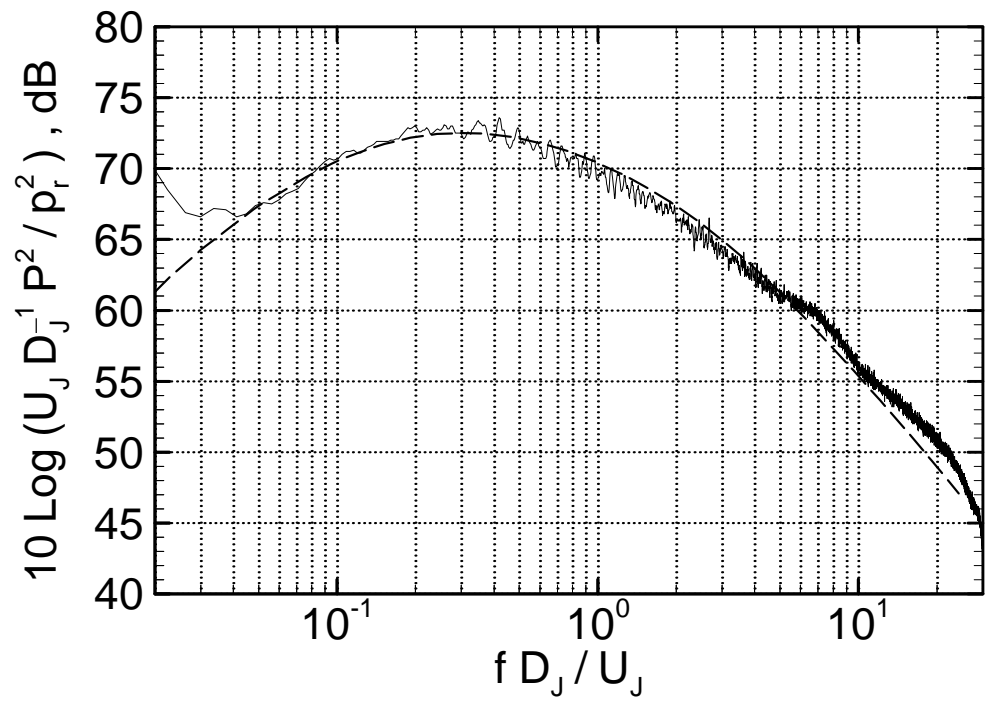

Figure 3.-Spectrum at $90^{\circ}$ and at $R / D_{\mathrm{J}}=100$ for a Mach 0.50 cold jet. Prediction (dashed line); data (solid line), $f \equiv \omega / 2 \pi, R \equiv|\boldsymbol{y}|$. 


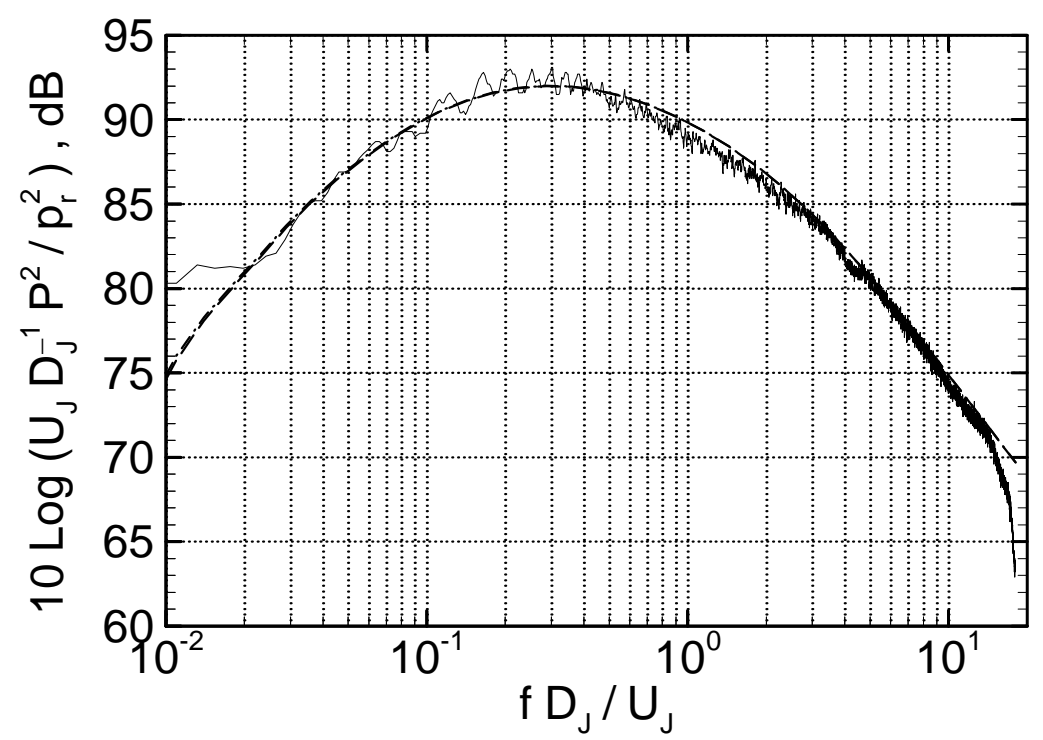

Figure 4.-Spectrum at $90^{\circ}$ and at $R / D_{J}=100$ for a Mach 0.90 cold jet. Prediction with $\kappa=0.0$ (dashed line); $\kappa=0.90$ (dash-dot); data (solid line), $f \equiv \omega / 2 \pi, R \equiv|y|$.

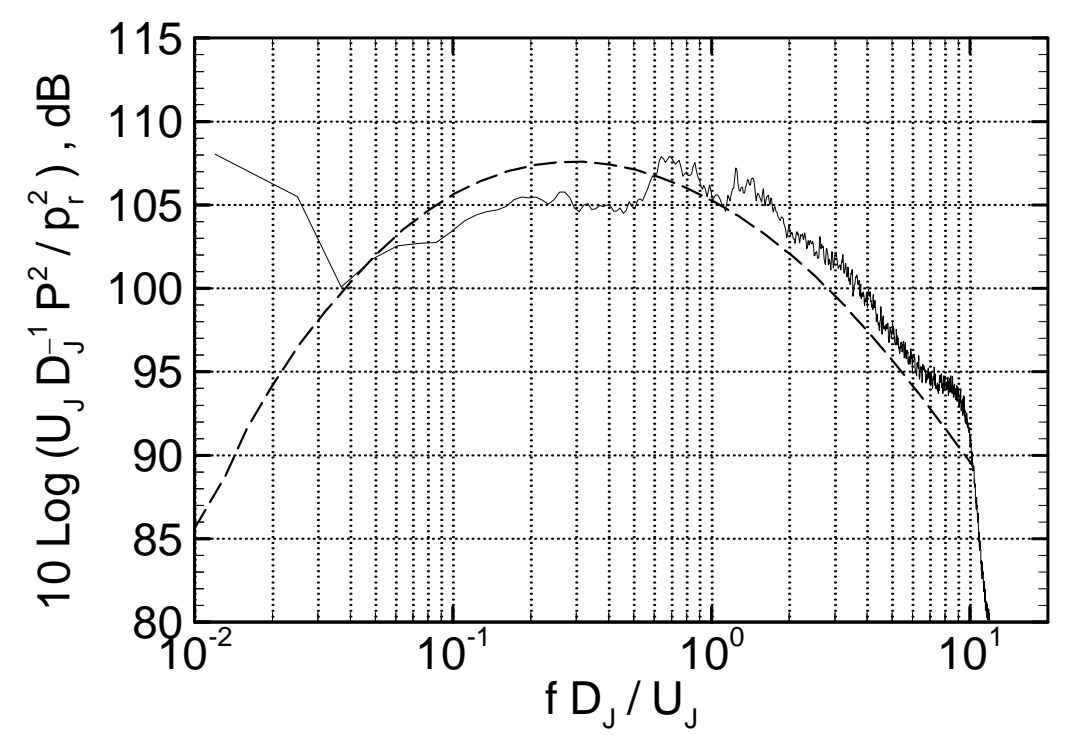

Figure 5.-Spectrum at $90^{\circ}$ and at $R / D_{J}=100$ for Mach 1.5 cold jet. Prediction (dash-dot); data (solid line), $f \equiv \omega / 2 \pi, R \equiv|\boldsymbol{y}|$. 


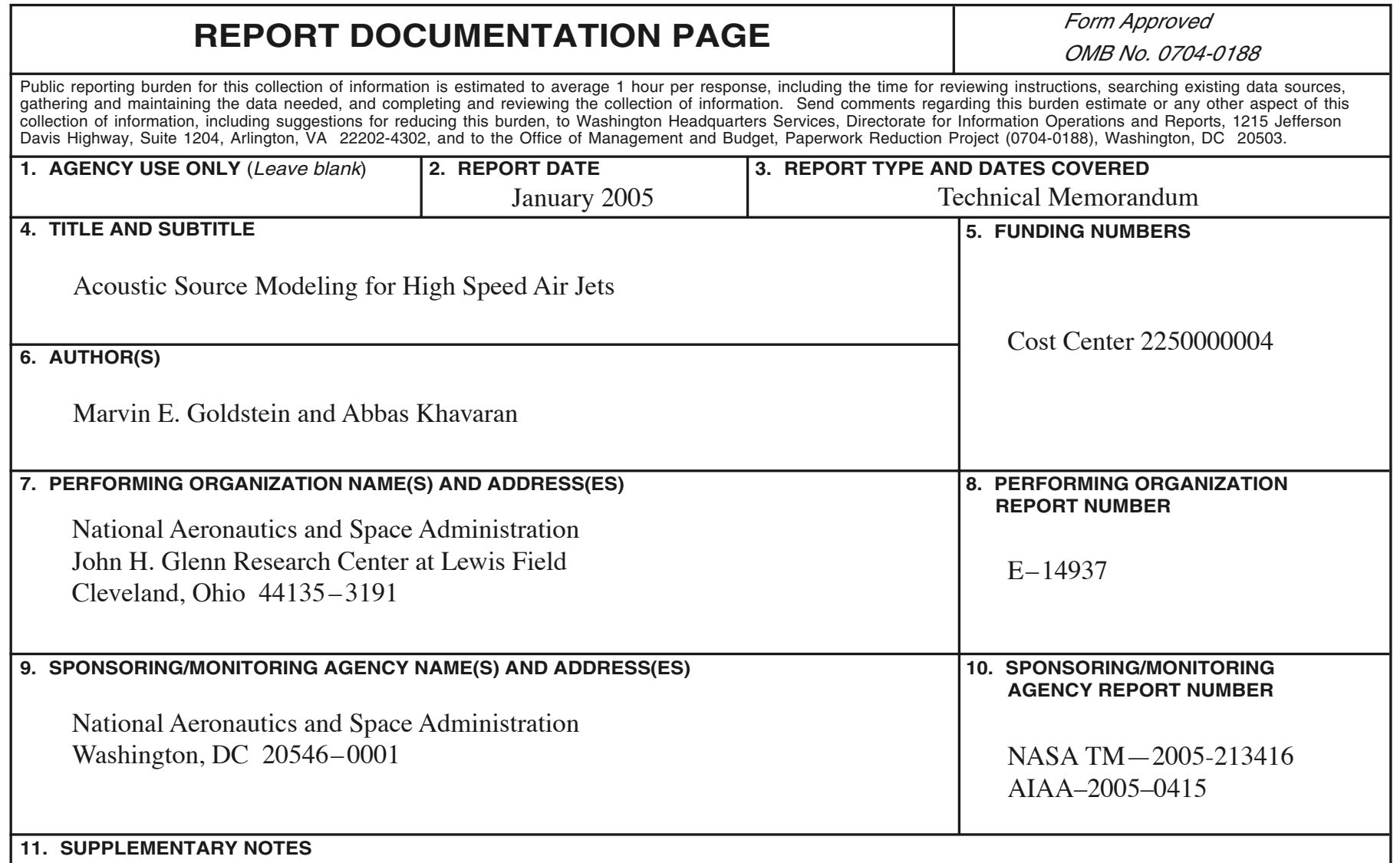

Prepared for the 43rd Aerospace Sciences Meeting and Exhibit sponsored by the American Institute of Aeronautics and Astronautics, Reno, Nevada, January 10-13, 2005. Marvin E. Goldstein, NASA Glenn Research Center; and Abbas Khavaran, QSS Group, Inc., 21000 Brookpark Road, Cleveland, Ohio 44135. Responsible person, Marvin E. Goldstein, organization code R, 216-433-5825.

12a. DISTRIBUTION/AVAILABILITY STATEMENT

12b. DISTRIBUTION CODE

Unclassified - Unlimited

Subject Categories: 01, 34, and 71

Distribution: Nonstandard

Available electronically at http://gltrs.grc.nasa.gov

This publication is available from the NASA Center for AeroSpace Information, 301-621-0390.

13. ABSTRACT (Maximum 200 words)

The far field acoustic spectra at $90^{\circ}$ to the downstream axis of some typical high speed jets are calculated from two different forms of Lilley's equation combined with some recent measurements of the relevant turbulent source function. These measurements, which were limited to a single point in a low Mach number flow, were extended to other conditions with the aid of a highly developed RANS calculation. The results are compared with experimental data over a range of Mach numbers. Both forms of the analogy lead to predictions that are in excellent agreement with the experimental data at subsonic Mach numbers. The agreement is also fairly good at supersonic speeds, but the data appears to be slightly contaminated by shock-associated noise in this case.

\begin{tabular}{|l|l|l|}
\hline $\begin{array}{l}\text { 14. SUBJECT TERMS } \\
\text { Jet noise; Turbulence; Acoustics }\end{array}$ \\
\hline $\begin{array}{c}\text { 17. SECURITY CLASSIFICATION } \\
\text { OF REPORT } \\
\text { Unclassified }\end{array}$ & $\begin{array}{c}\text { 18. SECURITY CLASSIFICATION } \\
\text { OF THIS PAGE } \\
\text { Unclassified }\end{array}$ & $\begin{array}{c}\text { 19. SECURITY CLASSIFICATION } \\
\text { OF ABSTRACT } \\
\text { Unclassified }\end{array}$ \\
\hline
\end{tabular}



